\title{
The Effects of Low-Intensity Therapeutic Ultrasound on Measurable Outcomes: A Critically Appraised Topic
}

\author{
Sarah Daniels, Gabriela Santiago, Jennifer Cuchna, and Bonnie Van Lunen
}

\begin{abstract}
Clinical Scenario: Therapeutic ultrasound (US) is a popular modality among health care professionals and is used to treat a variety of musculoskeletal conditions. A new technology has been established to allow for the miniaturization of the US unit. Patients receive treatment with the device secured to them, eliminating the portability constraint of traditional US units. Early studies suggest that this portable unit can deliver low-intensity acoustic energy achieving the same temperature increase and pain relief that come from traditional US units, in a more versatile and patient-friendly manner. Clinical Question: What effects does low-intensity therapeutic ultrasound (LITUS) have on measurable outcomes? Summary of Key Findings: The literature was searched for level 4 evidence or higher that investigated the effectiveness of LITUS. The literature search produced 3 possible studies related to the clinical question: 2 randomized controlled trials and 1 case series met the inclusion and exclusion criteria. Of the included studies, 1 study investigated the effects of LITUS on tissue temperature, 2 studies investigated the effects of LITUS on pain, and 1 study investigated LITUS effects on function. Clinical Bottom Line: The evidence supports the use of the LITUS unit to increase tissue temperature, decrease pain, and increase function. Therefore, practitioners may consider the use of the LITUS unit in patient populations over the use of the traditional high-intensity US treatment. Strength of Recommendation: In accordance with the 2009 Centre for Evidence-Based Medicine levels of evidence, there is grade I (insufficient) evidence to support the positive effects of the LITUS device for improving the following clinical outcomes: tissue temperature, decreasing pain, and increasing function. The inconsistency in the measured outcomes across the 3 studies only allows for minimal support of the LITUS device, warranting further research. Although clinical outcomes were different in each study, consistent evidence ranging from 4 to $1 \mathrm{~B}$ levels were found in the 3 included studies.
\end{abstract}

Keywords: temperature, pain, ultrasonic therapy

\section{Clinical Scenario}

Therapeutic ultrasound (US) is a popular modality among health care professionals and is used to treat a variety of musculoskeletal conditions. ${ }^{1}$ Throughout the past 70 years of clinical use, ${ }^{2}$ US has been shown to be an effective way to decrease pain,,${ }^{2,3}$ increase tissue temperature, ${ }^{4}$ and decrease tissue stiffness of trigger points, ${ }^{5}$ among other benefits. Treatment parameters with US are typically high intensity and occur over a short period (minutes). The US machine is large and stationary, forcing patients to be nonmobile for the duration of their treatment. However, the new technology established by Lewis et $\mathrm{al}^{3}$ has allowed for the miniaturization of the US unit. After pilot testing and trial runs, a low-intensity therapeutic ultrasound (LITUS) unit that fits easily into the palm of a hand has been developed and is currently available. ${ }^{2}$ To use the device, US coupling gel is applied directly to the skin over the desired treatment area and the transducer is secured with adhesive bandages. Patients receive treatment with the device secured to them, eliminating the portability constraint of traditional US units. Depending on the parameters chosen (refer to Table 2), the unit may provide treatment for up to 5-6 consecutive hours without needing to charge the unit battery. ${ }^{3}$ Early studies suggest that this portable unit can deliver low-intensity acoustic energy over a

Daniels and Santiago are with Post-Professional Athletic Training Program, Old Dominion University, Norfolk, VA. Cuchna is with Health Services Research PhD Program, Old Dominion University, Norfolk, VA. Van Lunen is with the School of Physical Therapy \& Athletic Training, Old Dominion University, Norfolk, VA. Cuchna (jcuch001@odu.edu) is corresponding author. prolonged period (hours), as well as medium-intensity treatments over a shorter period (minutes), achieving the same temperature increase and pain relief that come from traditional US units, in a more versatile and patient-friendly manner. ${ }^{3}$

\section{Focused Clinical Question}

What effects does LITUS have on measurable outcomes?

\section{Summary of Search, Best Evidence Appraised, and Key Findings}

- The literature was searched for studies of level 4 evidence or higher (based on levels of evidence ${ }^{6}$ ) that investigated the effectiveness of LITUS.

- The literature search produced 3 possible studies related to the clinical question: 2 randomized controlled trials and 1 case series met the inclusion and exclusion criteria $^{2-4}$ (Figure 1).

- Based on the design of the included studies, the modified Downs and Black checklist was used to critically appraise the methodological quality of each study. ${ }^{7}$ Two independent reviewers appraised each study and a consensus score was reached to determine the methodological quality. Studies with a score of $\geq 11 / 17$ were considered high methodological quality.

- Of the included studies, 1 study investigated the effects of LITUS on tissue temperature, ${ }^{4} 2$ studies investigated the 
Boolean phrases: low-intensity therapeutic ultrasound

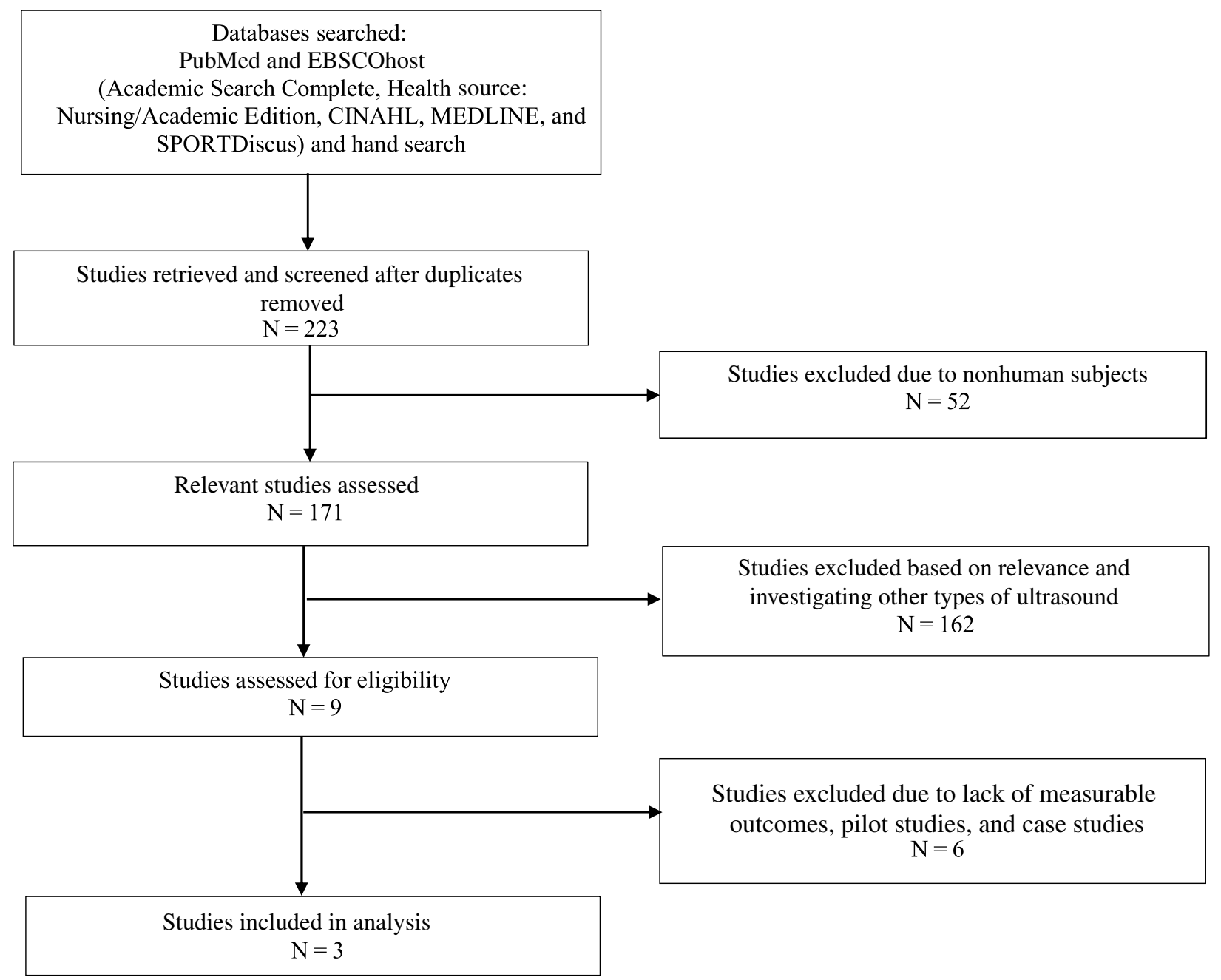

Figure 1 - Database search results.

effects of LITUS on pain, ${ }^{2,3}$ and 1 study investigated LITUS effects on function. ${ }^{2}$

- All included studies ${ }^{2-4}$ had positive effects for the measured outcomes.

\section{Clinical Bottom Line}

The evidence supports the use of the LITUS unit to increase tissue temperature, ${ }^{4}$ decrease pain, ${ }^{2,3}$ and increase function. ${ }^{2}$ Therefore, practitioners may consider the use of the LITUS unit in patient populations over the use of the traditional high-intensity US treatment.

\section{Strength of Recommendation}

In accordance with the 2009 Centre for Evidence-Based Medicine levels of evidence, there is grade I (insufficient) evidence to support the positive effects of the LITUS device for improving the following clinical outcomes: tissue temperature, ${ }^{4}$ decreasing pain, ${ }^{2,3}$ and increasing function. ${ }^{2}$ The inconsistency in the measured outcomes across the 3 studies only allows for minimal support of the LITUS device; therefore, more research must be conducted. Although clinical outcomes were different in each study, consistent evidence ranging from 4 to $1 \mathrm{~B}$ levels was found in the 3 included studies (Figure 2). ${ }^{2-4}$

\section{Search Strategy}

\section{Terms Used to Guide Search Strategy}

- Patient: Nonapplicable

- Intervention (or Assessment): Low-intensity therapeutic ultrasound

- Comparison: Nonapplicable

- Outcome: Nonapplicable

\section{Sources of Evidence Searched (Databases)}

- PubMed

- EBSCOhost

○ CINAHL

$\circ$ Health Source 


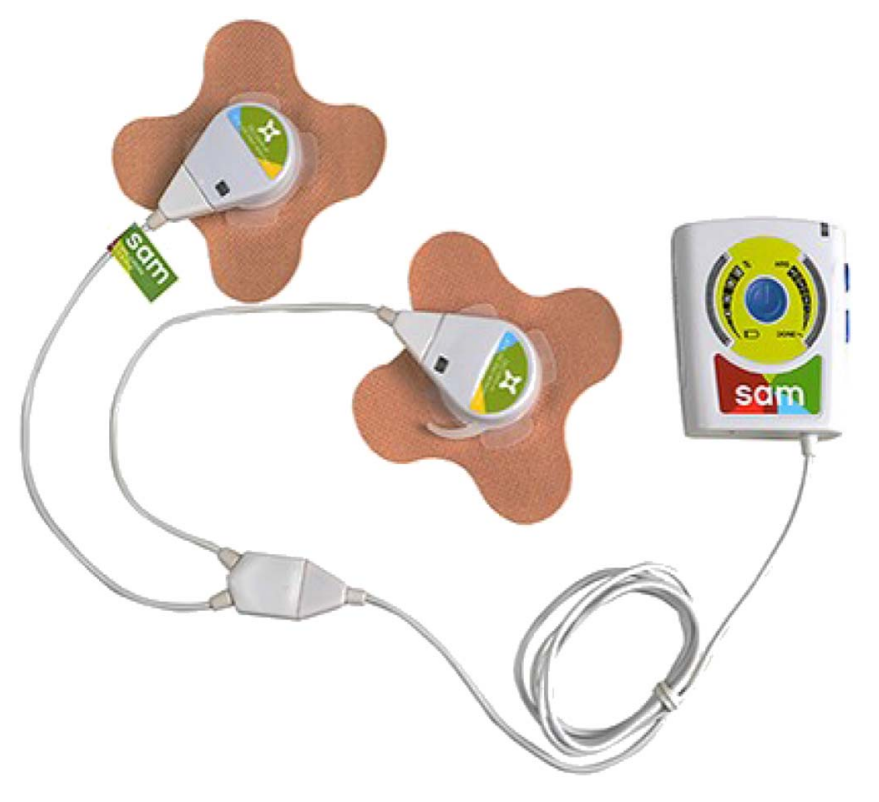

Figure 2 - Wearable LITUS device (sam®; ZetrOZ Inc, Trumbull, CT, USA).

\section{○ MEDLINE \\ - SPORTDiscus}

- Additional resources obtained via review of reference list and hand search.

\section{Inclusion and Exclusion Criteria (Including Search Limits)}

\section{Inclusion}

- Limited to English language studies

- Limited to level 4 evidence or higher

- Limited to the last 5 years (2012-2016)

- Limited to human subjects

- Prospective studies investigating and measuring outcomes of the LITUS device

\section{Exclusion}

- Studies with no formal article publications (eg, pilot studies, technical reports about the device)

\section{Results of Search}

Three relevant studies ${ }^{2-4}$ were identified and categorized as shown in Table 1 (based on Levels of Evidence, Oxford Centre for Evidence-Based Medicine, 2009).

\section{Best Evidence}

The studies in Table 2 were identified as the best evidence and selected for inclusion in this critically appraised topic. These studies were selected because they were graded with a level of evidence of 4 or higher and examined the effects of LITUS
Table 1 Summary of Study Designs of Articles Retrieved

\begin{tabular}{llcl}
\hline $\begin{array}{l}\text { Level of } \\
\text { evidence }\end{array}$ & Study design & $\begin{array}{c}\text { Number } \\
\text { located }\end{array}$ & Authors \\
\hline 1B & $\begin{array}{l}\text { Repeated-measures } \\
\text { cross-over design }\end{array}$ & 1 & Rigby et $\mathrm{al}^{4}$ \\
1B & $\begin{array}{l}\text { Randomized } \\
\text { control trial } \\
4\end{array}$ & 1 & Lewis et $\mathrm{al}^{3}$ \\
Case series & 1 & ${\text { Best et } \mathrm{al}^{2}}$ \\
\hline
\end{tabular}

on clinical outcomes (tissue temperature, pain, and patient function).

\section{Implications for Practice, Education, and Future Research}

All 3 studies ${ }^{2-4}$ appraised in this critically appraised topic identified positive results after use of a LITUS unit. Two studies ${ }^{3,4}$ were categorized as high-quality evidence (1B) due to the fact that they were randomized control trials, both receiving a score of 10/17 on the modified Downs and Black checklist. ${ }^{7}$ Sources of bias included lack of blinding of the therapists administering the therapy as well as assessors measuring the temperature at different time points. Although the Best et $\mathrm{al}^{2}$ study had the highest quality appraisal score $(15 / 17),{ }^{7}$ it was level 4 evidence due to the nature of the caseseries study design. The findings of this appraisal indicate that there is moderate-quality evidence to suggest that treatment from a wearable LITUS unit increases tissue temperature, decreases pain, and increases function.

Although the findings were consistent, the studies had notable differences. Both Rigby et $\mathrm{al}^{4}$ and Lewis et $\mathrm{al}^{3}$ enrolled patients who were randomly assigned to a treatment or placebo (control) group. Participants were blinded to which group they were in, and both studies had a $100 \%$ completion rate with no adverse reactions reported. Best et $\mathrm{al}^{2}$ had no control group in their study and their participants were recruited based on having one of the 2 conditions: Achilles or elbow tendinopathy. All of the patients received the intervention, and only 16 of the initial 25 enrolled completed the full 6 -week protocol. ${ }^{2}$ Having such a high dropout rate reduced the already small sample size, decreasing the external validity of the study as well as limiting the generalizability of the results to people with only Achilles or elbow tendinopathies.

Additional variation in the studies included each study having a different implementation of their chosen intervention. Rigby et $\mathrm{al}^{4}$ had participants attend 2 test sessions separated by 48 hours, during which all data collection was completed. Lewis et $\mathrm{al}^{3}$ conducted 1-hour treatment sessions for 10 consecutive days, while Best et $\mathrm{al}^{2}$ enrolled participants in a 6 -week protocol with treatment 4 hours per day at least 5 times per week. Implementing each intervention in a different way eliminates continuity of the studies, and makes it impossible to compare interventions and results across studies. This allows for us to examine each study individually for effectiveness of intervention, but disallows an overall generalization of the results to be made.

In addition to the different enrollment methods, the included studies had different outcome measures as well. Rigby et $\mathrm{al}^{4}$ assessed tissue temperature of the triceps surae muscle using microprobe thermocouples. The Food and Drug Administration- 
Table 2 Characteristics of Included Studies

\begin{tabular}{|c|c|c|}
\hline & Rigby et al $^{4}$ & Lewis et $\mathrm{al}^{3}$ \\
\hline Study design & Repeated-measures cross-over design & Randomized control trial \\
\hline Participants & $\begin{array}{l}\text { A total of } 26 \text { healthy participants [ } 16 \text { men } \\
\text { and } 10 \text { women, age }=23.0(2.1) \text { y] } \\
\text { enrolled and completed the study. } \\
\text { Exclusion criteria: subjects presenting with } \\
\text { fever, lower leg infection, or open wound; } \\
\text { compromised circulation or sensation; } \\
\text { injury to the lower leg within the } 2 \text { mo } \\
\text { before study or contraindication to US. } \\
\text { Groups were unbalanced intentionally to } \\
\text { assess intramuscular temperature changes } \\
\text { produced by the LITUS device while } \\
\text { controlling possible environmental } \\
\text { variables with a placebo group. }\end{array}$ & $\begin{array}{l}\text { A total of } 30 \text { patients were recruited and } \\
\text { randomly assigned to the placebo ( } \mathrm{n}=10 \text { ) } \\
\text { and active }(\mathrm{n}=20) \text { conditions. } \\
\text { Active group: } \mathrm{n}=10 \text { males and } \mathrm{n}=10 \\
\text { females; placebo group: } \mathrm{n}=1 \text { male and } \\
\mathrm{n}=9 \text { females. } \\
\text { Inclusion criteria: age }=40-60 \mathrm{y} \text {; not be } \\
\text { pregnant; willing and able to self- } \\
\text { administer Tx daily; physician clearance; } \\
\text { agreement to record any reduction or } \\
\text { increase in prescription drug use in daily } \\
\text { diary; VAS scores of } 4-7 \text {. } \\
\text { Exclusion criteria: neuropathy; } \\
\text { pregnancy; prisoners; surgery in the target } \\
\text { area within the last } 6 \text { mo; nonambulatory; } \\
\text { increasing use or initiate new use of meds } \\
\text { during the study; used topical agents other } \\
\text { than the US gel provided; clinically } \\
\text { significant or unstable medical or } \\
\text { psychological condition; participated in a } \\
\text { clinical trial for an investigational drug } \\
\text { and/or agent within } 30 \mathrm{~d} \text { before screening; } \\
\text { injury-related litigation in the target area; } \\
\text { clinically significant abnormal neurologic } \\
\text { Hx or examination at screening; back } \\
\text { spasms related to major trauma or } \\
\text { work-related injury; other severe pain that } \\
\text { may have confounded assessment or } \\
\text { self-evaluation of the trapezius myalgia. }\end{array}$ \\
\hline
\end{tabular}

Intervention investigated
Participants attended 2 test sessions separated by $48 \mathrm{~h}$ for Tx with the LITUS device (model sam-12; ZetrOZ Inc). During first visit, the participants received Tx with either one $7000 \mathrm{~J}$ or $14,000 \mathrm{~J}$ US transducer, and alternate $\mathrm{Tx}$ administered at second testing session. Parameters were set to $3-\mathrm{MHz}$ frequency and $0.132 \mathrm{~W} / \mathrm{cm}^{2}$ spatial average temporal average intensity for $3 \mathrm{~h}$.

Tx occurred at the same time of the day to limit diurnal variation. Participants in the placebo group received the placebo Tx for both sessions. LITUS devices were configured and applied. Devices were clipped to a medical tape bandage and US gel was used as coupling agent. For singletransducer Tx, devices were equally spaced above previously placed temperature probes within the tissue; for dual-transducer treatments, devices were spaced above the temperature probes. Participants were then instructed to lie still for remainder of treatment (180 $\mathrm{min})$ FDA status of the device was not clearly stated by authors.
Participants participated in at least ten 1-h US Tx sessions at the onset of heightened pain caused by trapezium spasm. Tx parameters were not identified by authors, but the LITUS device works at $2.5-3 \mathrm{MHz}$ with $0.03-2 \mathrm{~W} / \mathrm{cm}^{2}$ intensity capability for $0.3-18 \mathrm{~h}$ of treatment, depending on the output setting.

Sessions lasted $1 \mathrm{~h} / \mathrm{d}$ for continuous $10 \mathrm{~d}$; participants were instructed to record their pain level every 15 min during duration of Tx. Participants were instructed how to properly apply and utilize the LITUS device, and then given individual participant kit, which included the LITUS device with a belt clip, three 2-oz US coupling gel packets, twenty $10 \times 10.5-\mathrm{cm}$ adhesive bandages, a system wall charger, a user manual, and a patient diary for self-reporting. Majority of the participants completed the study from their home or work environment, minimizing the impact the device had on their lifestyle.

LITUS device manufacturer information was not provided, but the electronics and battery pack were integrated into a commercially available housing (OKW, Bridgeville, PA). FDA status of the device was not clearly stated by authors.

\section{Best et $\mathrm{al}^{2}$}

Case series

A total of 25 participants with Achilles $[\mathrm{n}=5$, age $=61.6(8.0)$ y] or elbow $[\mathrm{n}=20$, age $46.9(9.6)$ y] tendinopathy were enrolled.

Inclusion criteria: age $=18-65 \mathrm{y}$; able to shave/remove hair in the area where the applicator of the device will be applied; access to a mobile phone or camera to take a picture of the treatment site after use of device; $\mathrm{BMI} \leq 30.0$; no NSAIDs treatment or prescription pain meds at enrollment, and agree to document all pain meds used during the study period.

Exclusion criteria: Hx or current Dx of a tendon tear; known neuropathy; prisoners; smokers; type 1 or type 2 diabetes; surgery in target Tx area within the last $6 \mathrm{mo}$; nonambulatory; pacemaker; other malignancy; use of pain meds during the trial unless medically necessary to ensure safety; use of topical agents other than the US gel provided; refuse to discontinue all other interventional treatment modalities; underwent a local corticosteroid or platelet-rich plasma injection within the past $3 \mathrm{mo}$; medical or psychological condition that would compromise participation; participated in a clinical trial for an investigational drug and/or agent within $30 \mathrm{~d}$ prior to screening; involved in any injury-related litigation; open sores or wounds in the Tx preventing device usage.

Subjects recruited from 3 clinics and compensated up to $\$ 150$ based on the number of completed study visits. Three subjects withdrew from study. Twentyone participants completed the first follow-up visit at $2 \mathrm{wk}, 18$ completed $4 \mathrm{wk}$, and 16 completed the full 6 -wk protocol.

All subjects reported to the clinic initial screening and enrollment visit. Subjects taught to apply the LITUS device ( $\mathrm{sam}^{\circledR}$; ZetrOZ Inc, cleared by FDA in Nov 2013) and instructed to apply it to their affected tendon for 4-h treatment sessions at least 5 times per wk for $6 \mathrm{wk}$. Parameters of US Tx were 3-MHz frequency and $0.132 \mathrm{~W} / \mathrm{cm}^{2}$ intensity per applicator, continuous wave form, BNR: $<5: 1$, ERA: $6 \mathrm{~cm}^{2}$ (per applicator) for up to $4 \mathrm{~h}$ per treatment session.

Subjects returned to the clinic for assessments of safety and efficacy at the end of 2, 4, and 6 wk of Tx, and maintained a diary of Tx and symptoms at home throughout the 6-wk period. Each subject wore 1 applicator directly over the tendon (Achilles or medial/lateral elbow) and another just upstream of it using a Y-adaptor connected to the power controller. 
Table 2 (continued)

\begin{tabular}{ll}
\hline & Rigby et al $^{\mathbf{4}}$ \\
\hline Outcome & Tissue temperature was assessed using \\
measures & microprobe thermocouple (MT 23/5; \\
& Physitemp Instruments LLC, Clifton, NJ). \\
& Two PT-6 thermocouples (Physitemp \\
& Instruments LLC) were used to assess skin \\
& surface and ambient temperature. Initial \\
& tissue temperature was measured 5 min \\
& before the 180-min LITUS treatment, and \\
& posttreatment temperature was recorded \\
& for 30 min.
\end{tabular}
for $30 \mathrm{~min}$.

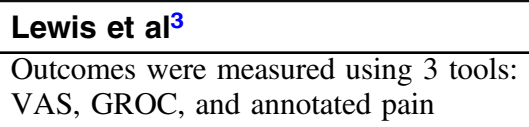

diagrams. Patients reported pain before and after Tx.

Patients successfully reported with all 3 measures. VAS results among patients were positive with the greatest pain reduction reported during the first 3-4 d. Pain reduction was $8 \%$ for placebo user, $12 \%$ for active female users, and $19 \%$ for active male users. Across the $10 \mathrm{Tx}$ sessions, GROC scores showed improvement $60 \%$ greater than the placebo group. Active female patients reported no statistically significant or trending effects, whereas the placebo group on average reported upper back pain after $60 \mathrm{~min}$ of Tx. Active men reported a $30 \%$ pain reduction over placebo. Active male and female patients responded to treatment faster than the placebo group. Post-60-min LITUS Tx, placebo and active male groups reported increased pain, while active females reported a continued decrease in pain after conclusion of the treatment.

1B

Modified Downs and Black Score 10/17

The LITUS device in conjunction with pain management medication is effective at reducing patients' chronic trapezius myalgia while producing no reports of negative side effects.

\section{Best et $\mathrm{al}^{2}$}

Pain ratings were recorded using a standardized 11-point numeric rating scale. Subjects self-reported pain 4 times per day during 6-wk Tx: before Tx, $30 \mathrm{~min}$ into $\mathrm{Tx}, 2 \mathrm{~h}$ into $\mathrm{Tx}$, and at conclusion of the Tx. Subjects rated their pain using the numeric rating scale providing a rating for the worst and best levels of pain for the previous week and current level of pain. Force generation and grip strength were assessed at each study visit via a handheld dynamometer for elbow pathology and MicroFET dynamometer for Achilles pathology.

Of the subjects who completed the full 6-wk protocol, $62.5 \%$ experienced at least a $50 \%$ decrease in pain, and on average had a 3.94 (2.15) point decrease from baseline. At the beginning of the experiment, grip strength was 26.88 (9.89) $\mathrm{kg}$ force in the injured arm and 33.49 (11.15) $\mathrm{kg}$ force in the uninjured arm. Grip strength increased by $2.83(5.52) \mathrm{kg}$ force between baseline and week 2 in the injured arm. No significant increases in strength in the untreated arm. On average, there was improvement in exerted force measured by dynamometer of the affected leg with treatment in the Achilles group. Statistical analyses on the pain and strength data were not performed due to the limited sample size.

3B

Modified Downs and Black Score 15/17

Sustained acoustic medicine may be an effective therapy for elbow and Achilles tendinopathy. Results provide evidence that the LITUS device safely treats tendon injuries.

Abbreviations: BNR, beam nonuniformity ratio; BMI, body mass index; Dx, diagnosis; ERA, effective radiating area; FDA, Food and Drug Administration; GROC, global rating of change; Hx, history; LITUS, low-intensity therapeutic ultrasound; IM, intramuscular; NSAID, nonsteroidal anti-inflammatory drug; Tx, treatment; US, ultrasound; VAS, Visual Analog Scale Score.

approved LITUS device (model sam-12; ZetrOZ Inc, Trumbull, CT) was set to a frequency of $3 \mathrm{MHz}$ and an intensity of $0.132 \mathrm{~W} /$ $\mathrm{cm}^{2}$ for a total of 3 hours. ${ }^{4}$ Temperature was measured 5 minutes prior to the treatment and for 30 minutes after the 3-hour treatment session. Temperatures were taken in degree Celsius, and average temperature increases between $3^{\circ} \mathrm{C}$ and $4^{\circ} \mathrm{C}$ were found for each treatment condition when compared with the control group. These increases $\left(3^{\circ} \mathrm{C}-4^{\circ} \mathrm{C}\right)$ are very similar to temperature increases achieved by traditional US units. ${ }^{3}$

Lewis et $\mathrm{al}^{3}$ assessed pain in patients with chronic trapezius myalgia using 3 different outcome measures: the Visual Analog Scale Score, global rating of change, and annotated pain diagrams. Visual Analog Scale Score results among all patients were positive, with the majority of patients reporting the greatest reduction in pain during the first 3-4 days. On average, placebo users had an $8 \%$ pain reduction; female active users had a $12 \%$ pain reduction, and male active users had a 19\% pain reduction. The average global rating of change score in the test group reported improvements $60 \%$ greater than that reported in the placebo group. Findings suggest that the LITUS unit is effective at reducing chronic trapezius myalgia while producing no reports of negative side effects. However, the majority of participants were previously using a prescribed method of pain control and they were not asked to stop taking their medication while participating in the study, making it difficult to determine whether the results were due to the LITUS unit, the pain medications, or a combination of the 2 interventions.

Best et $\mathrm{al}^{2}$ measured pain and function of participants with either chronic elbow or chronic Achilles pathology initially, and at 
the end of 2, 4, and 6 weeks of treatment. Pain was measured using a standardized, 11-point numeric rating scale: 0 (no pain) and 10 (extreme pain). Function was measured by taking musculotendinous force generation assessments at each visit. Elbow pathology subjects measured grip strength with a handheld dynamometer, while Achilles pathology subjects used a MicroFET dynamometer (Hoggan Scientific LLC, Salt Lake City, UT). Of the subjects in the elbow tendinopathy group who completed the full 6-week protocol, $62.5 \%$ experienced at least a $50 \%$ decrease in pain. Grip strength increased by $2.83(5.52) \mathrm{kg}$ force between baseline and week 2 in the injured arm. On average, there was an improvement in exerted force measured by the dynamometer of the affected leg with treatment in the Achilles group. Results suggest that sustained acoustic medicine may be an effective therapy for elbow and Achilles tendinopathy.

To date, there are the only 3 studies $^{2-4}$ to investigate the effects of the LITUS unit on measurable outcomes. This portable lightweight unit has produced increased tissue temperatures and pain relief comparable with the effects achieved by traditional highintensity, short-duration therapeutic US. ${ }^{3}$ The battery pack of the LITUS unit is small enough to fit in your hand, and the transducers attach easily to the skin for the duration of the treatment session. Furthermore, the treatment process is simple enough that patients can be instructed by clinicians on application during their first usage, and should be able to apply and treat themselves for the remaining duration of their treatment sessions. This allows clinicians to spend their time working with other patients, as well as allowing the patient the ability to carry out their activities of daily living while wearing the unit. In addition, patients will be able to self-administer treatments as they deem necessary, eliminating the need for multiple scheduled appointments with their clinicians just for treatment.

Future research is needed to examine the long-term effects of the LITUS unit, and to establish any true differences between the portable LITUS unit and a traditional high-intensity, short-duration US unit. Conducting future studies over time may demonstrate how the long-duration, low-intensity acoustic energy affects different tissues, as well as tissue degradation rates when compared with those seen with traditional US. Identifying the influence of the LITUS unit on different measures of self-reported function (regional, global, and mental) may also provide valuable information to support the use of the wearable LITUS unit. Finally, designing future studies using good-quality randomized controlled trials will help provide higher-level evidence to support the use of a wearable LITUS unit. This critically appraised topic should be reviewed in 2 years to determine whether there is additional best evidence that may change the clinical bottom line for this clinical question.

\section{References}

1. Draper DO, Castel JC, Castel D. Rate of temperature increase in human muscle during $1 \mathrm{MHz}$ and $3 \mathrm{MHz}$ continuous ultrasound. J Orthop Sports Phys Ther. 1995;22(4):142-150. PubMed doi: 10.2519/jospt.1995.22.4.142

2. Best TM, Moore B, Jarit P, Moorman CT, Lewis GK. Sustained acoustic medicine: wearable, long duration ultrasonic therapy for the treatment of tendinopathy. Phys Sportsmed. 2015;43(4):366-374. PubMed doi:10.1080/00913847.2015.1095617

3. Lewis GK, Langer MD, Henderson CR Jr, Ortiz R. Design and evaluation of a wearable self-applied therapeutic ultrasound device for chronic myofascial pain. Ultrasound Med Biol. 2013;39(8): 1429-1439. PubMed doi:10.1016/j.ultrasmedbio.2013.03.007

4. Rigby JH, Taggart RM, Stratton KL, Lewis GK Jr, Draper DO. Intramuscular heating characteristics of multihour low-intensity therapeutic ultrasound. J Athl Train. 2015;50(11):1158-1164. PubMed doi:10.4085/1062-6050-50.11.03

5. Draper DO, Mahaffey C, Kaiser D, Eggett D, Jarmin J. Thermal ultrasound decreases tissue stiffness of trigger points in upper trapezius muscles. Physiother Theory Pract. 2010;26(3):167-172. PubMed doi:10.3109/09593980903423079

6. Phillips B, Ball C, Sackett D, et al. Oxford Centre for Evidence-based Medicine - Levels of Evidence (March 2009). Oxford Centre for Evidence-Based Medicine. https://www.cebm.net/2009/06/oxfordcentre-evidence-based-medicine-levels-evidence-march-2009/

7. Munn J, Sullivan SJ, Schneiders AG. Evidence of sensorimotor deficits in functional ankle instability: a systematic review with metaanalysis. J Sci Med Sport. 2010;13:2-12. PubMed doi:10.1016/ j.jsams.2009.03.004 